\title{
Effect of Spinal Stabilization with Visual Feedback on the Balance of Chronic Stroke Patients
}

\author{
Jung Byung Chae, PT, PhD ${ }^{1)}$, MoOn Hwan LeE, PT, PhD²) \\ 1) Department of Physical Therapy, Masan University \\ ${ }^{2)}$ Department of Physical Therapy, International University of Korea: San 270, Sangmun-Ri, Munsan- \\ Eup, Jinju City, 660-759, Republic of Korea. TEL +82 55-751-8292, FAX: +82 55-751-8299, \\ E-mail: serhan0520@daum.net
}

\begin{abstract}
Purpose] This study aimed to find the effect of spinal stabilization with visual feedback on the balance of stroke survivors. [Subjects and Methods] Twenty-one chronic stroke patients participated in the study. The experimental group underwent a spinal stabilization exercise program 30 minutes per day, 5 times per week, for a total of 8 weeks. The control group received conventional physiotherapy. The Berg balance scale, and the Timed up and go and Functional reach tests were performed to assess subjects's balance. [Results] There were significant differences between before and after the intervention in all balance parameters of the experimental group, but the control group showed no significant differences. A statistically significant difference was found between the two groups for the Timed up-and-go test. [Conclusion] The results of this study show that spinal stabilization with visual feedback is an effective intervention for improving the balance of chronic stroke patients.
\end{abstract}

Key words: Spinal stabilization, Balance, Stroke

(This article was submitted Aug. 31, 2010, and was accepted Sep. 26, 2010)

\section{INTRODUCTION}

Stroke, which is the most common cerebral circulation disorder, is one of the 3 major causes of death among humans along with malignant tumors and heart attacks ${ }^{1)}$. In South Korea, it holds the second place after malignant tumors in the list of causes of death in the elderly population over 65 years of age ${ }^{2)}$.

Neurological and functional recovery in most stroke survivors reach their peak within 5 months after the onset of stroke, and some researchers even argue that no further improvements can be expected at all after that point ${ }^{3}$. However, there are also researchers who have reported that aerobic capacity and balance can be improved through exercise training even in chronic hemiparetic stroke patients $^{4-8)}$.

In order to allow stroke patients to maintain adequate balance according to changing environments, appropriate muscle power and endurance of the spinal muscles is very important. This is because the abdominal muscles and the spinal muscles are closely related to spinal stability and their function is essential in spinal movements and posture control $^{9)}$.

Improvement in the muscle power and endurance of these spinal muscles is possible through spinal stabilization exercise training, the application of which is currently limited to lower back pain patients. Spinal stabilization exercise training is an exercise that trains the transverse abdominis and multifidus simultaneously, and it is known to be effective for improving unstable posture maintenance and control ${ }^{10)}$.

Furthermore, spinal stabilization exercise training can also be safely applied to stroke patients who cannot walk easily since it can be performed in the sitting position. It can also lessen risk factors of exposure to secondary problems such as falls, but up till now its use has been largely limited to lower back pain patients.

There are few reports in the literature about the effect on the balance of stroke patients of spinal stabilization exercise training with visual feedback, which is currently only administered to lower back pain patients, for stroke patients and studied the effect on their balance.

\section{SUBJECTS AND METHODS}

The subjects of this study were 21 stroke patients receiving physiotherapy who understood the contents of this study and agreed to participate voluntarily. The subjects were randomly divided into experimental (male 7, female 2) and control group (male 7, female 5). The average age was 56.33 years, the average height was $166.55 \mathrm{~cm}$, and the average weight was $64.66 \mathrm{~kg}$ in the experimental group, and in the control group the average age was 56.77 years, average height was $164.66 \mathrm{~cm}$, and the average weight was $64.44 \mathrm{~kg}$. The time since onset of stroke was 21.44 month ( $\pm 6.85)$ in the experimental group, and 21.55 months ( \pm 
6.24) in the control group. No significant differences were found between the two groups when a test of homogeneity was performed on the two groups $(p>0.05)$.

The visual feedback exercise performed by the experimental group uses a Biofeedback Unit Stabilizer. The exercise program consists of abdominal muscle reeducation in the four-foot kneeling position, curl exercise in the prone position, and abdominal muscle re-education in the righting position. The participants were instructed to perform isometric contractions of the transverse abdominis and multifidus muscles.

First, abdominal muscle re-education in the four-foot kneeling position was carried out repetitions of abdominal wall drawing and drift were performed to promote deep muscle activation to enable the trunk and pelvic area to maintain its normal curvature. Next, in the prone position, the pressure sensor of the Biofeedback Unit Stabilizer was placed on the patient's inferior abdomen, on the line connecting the right and left anterior superior iliac spines (ASIS). Then the pressure gauge on the monitor of the Biofeedback Unit Stabilizer was inflated up to $70 \mathrm{mmHg}$. The patients were instructed to perform drawing movements of the lower abdomen, so that the pressure gauge on the monitor would decrease about $6-8 \mathrm{mmHg}$. A maximal pressure decrease up to $10 \mathrm{mmHg}$ was allowed. Abdominal muscle re-education in the righting position was done in sequential steps, in the sitting position and the upright position, and the patients were instructed to perform cocontraction of the transverse abdominal muscle and multifidus muscles.

The exercise was carried out after a specially trained therapist explained and demonstrated the exercise to the participants so that they would fully understand. It was performed for 30 minutes per day, and the patients were allowed to take some rest before continuing the exercise when they felt fatigue or discomfort. The control group underwent conventional physiotherapy. After 8 weeks of therapeutic intervention, all the subjects were examined to ascertain the effects of therapeutic intervention in each group. Balance was assessed pre- and post-intervention using the Berg Balance Scale (BBS), the Timed Up-and-Go Test (TUG), and the Functional Reach Test (FRT).

The data collected from 9 patients in the experimental group and 12 patients in the control group in this study were analyzed using the statistics program, SPSS (version 14.0). The paired sample t-test was performed for pre- and post- intervention significance tests in each group. The independent t-test was performed for significance tests between the two groups. The significance level was chosen as 0.05 .

\section{RESULTS}

The BBS of the experimental group and the control group before and after the intervention were compared. There was a significant improvement in the post-test results of the experimental group $(\mathrm{p}<0.05)$, whereas the control group did not show any significant change $(\mathrm{p}>0.05)$. In the pre- and post-intervention mean differences comparison, there was a significant difference between the two groups $(\mathrm{p}<0.05)$ (Table 1).

The TUG results of the experimental group and the control group before and after the intervention were compared. There was a significant decrease in the postintervention results of the experimental group $(p<0.05)$, whereas the control group did not show any significant change $(p>0.05)$. In the pre- and post-intervention mean differences comparison, there was a significant difference between the two groups $(\mathrm{p}>0.05)$ (Table 1$)$.

The FRT results of the experimental group and the control group before and after the intervention were compared. There was a significant increase in the postintervention results of the experimental group $(p<0.05)$, whereas the control group did not show any significant change $(p>0.05)$. In the pre- and post-intervention mean differences comparison, there was no significant difference between the two groups $(\mathrm{p}>0.05)$ (Table 1$)$.

\section{DISCUSSION}

This study examined 21 chronic stroke patients who had a stroke incident more than 6 months before the study began. Nine patients (experimental group) completed a spinal stabilization exercise program of 30 minutes per day, 5 times per week, for 8 weeks. These patients showed significant changes in BBS, TUG, and FRT results after the intervention. These results suggest that even for chronic stroke patients who had a stroke incident more than 6 months ago and whose brain spasticity would have stopped, exercise training is effective for improving their balance ability.

Similar results have also be seen in previous studies.

Table 1. A comparison of balance measures between pre- and post-intervention in each group

\begin{tabular}{lrccc}
\hline Parameter & Group & Pre-intervention & Post-intervention & Mean difference \\
\hline BBS $<$ score $>$ & Exp. Group & $48.22 \pm 1.20$ & $49.77 \pm 1.85$ & $1.55 \pm 1.87^{\dagger}$ \\
& Cont. Group & $48.66 \pm 1.22$ & $48.77 \pm 0.85$ & $0.11 \pm 0.33$ \\
\hline TUG $<$ sec $>$ & Exp. Group & $31.68 \pm 11.94$ & $30.41 \pm 11.95$ & $-1.27 \pm 1.43^{* \dagger}$ \\
& Cont. Group & $23.96 \pm 13.00$ & $24.24 \pm 12.72$ & $0.28 \pm 0.89$ \\
\hline FRT $<$ cm $>$ & Exp. Group & $7.22 \pm 3.00$ & $8.08 \pm 3.19$ & $0.86 \pm 1.42^{*}$ \\
& Cont. Group & $7.07 \pm 2.19$ & $7.16 \pm 2.00$ & $0.08 \pm 0.70$ \\
\hline
\end{tabular}

Abb. BBS: Berg balance scale; TUG: timed up-and-go test; FRT: functional reach test. $* \mathrm{p}<0.05 .{ }^{*}$ significant difference between pre- and post-intervention in each group. ${ }^{\dagger}$ significant difference between experimental and control groups. 
Sackley and Baguly ${ }^{4}$ measured the distribution of weight load on both legs of 90 stroke patients in the standing position in order to study the relationship between the degree of distribution, motor function, and the independence of everyday movements. They found that there was a significant positive correlation between motor function and activities of daily living with asymmetric weight load. Shumway-Cook et al. ${ }^{5)}$ conducted 2 weeks of weight-shift training with visual feedback for 8 of 16 hemiparetic patients. They found that the symmetry of weight load on both legs significantly increased in the experimental group compared to the control group.

Furthermore, Srivastava et al. ${ }^{6)}$ conducted visual feedback balance training using force plates with 45 chronic stroke patients and reported that the patients' balance and ambulation capabilities improved significantly after the intervention. Weiss et al. ${ }^{7)}$ reported increased leg muscle power, balance, and mobility after 12 weeks of gradual resistance exercise training using $70 \%$ weight of $1 \mathrm{RM}$ of leg muscle. A recent study by Bastille and Gill-Body, ${ }^{8)}$ reported that mobility and balance was improved after chronic stroke patients at more than 9 months after onset performed yoga for 1 hour per 2 times per week for 8 weeks.

All exercises share the characteristic of showing a decrease in their effects after a certain point. Since the effect of the exercise will ultimately disappear, it is necessary to modify the exercise intensity at an adequate time or to modify the pattern or program of the exercise according to patients' physical abilities. From this point of view, this study had the limitation of having implemented a uniform course of spinal stabilization exercises for 8 weeks without any modification during the program. Also, the number of subjects was too small to generalize the results of this study to other chronic stroke patients. Therefore, future studies should involve a larger number of subjects as well as determine what level of intensity and duration is more effective for improving the balance and mobility of stroke patients.

Despite the limitations of this study, the results show that spinal stabilization with visual feedback had a positive effect on the balance of stroke patients. Therefore, for an effective intervention that can improve patients' balance, physicians who treat stroke patients should not only conduct training that stimulates the proprioceptors of patients' soles and leg joints, but should also implement spinal stabilization with visual feedback for patients who have difficulties in the standing position or in all stages of standing training. This study provides data that will serve as objective evidence for physicians who treat stroke patients in clinics.

\section{REFERENCES}

1) Michael KM, Allen JK, Macko RF: Reduced ambulatory activity after stroke: The role of balance, gait, and cardiovascular fitness. Arch Phys Med Rehabil, 2005, 86: 1552-1556.

2) Korea National Statistical Office. 2009.

3) Jorgensen HS, Nakayama H, Raaschou HO, et al.: Outcome and time course of recovery in stroke, part II: time course of recovery. The Copenhagen Study. Arch Phys Med Rehabil. 1995, 76: 406-412.

4) Sackley CM, Baguly BI: Visual feedback after stroke with balance performance monitor: two single case studies. Clin Rehabil, 1993, 7: 189195.

5) Shumway-Cook A, Anson D, Haller S: Postural sway biofeedback its effect on reestabilishing stance stability in hemiplegic patients. Arch Phys Med Rehabili, 1988, 69: 395-400.

6) Srivastava A, Taly AB, Gupta AG, et al.: Post-stroke balance training: role of force platform with visual feedback technique. J Neurol Sci, 2009, 287: 8993.

7) Weiss A, Suzuki T, Bean J, et al.: High intensity strength training improves strength and functional performance after stroke. Am J Phys Med Rehabil, 2000, 79: 369-376.

8) Bastille JV, Gill-Body KM: A yoga-based exercise program for people with chronic poststroke hemiparesis. Phys Ther, 2004, 84: 33-48.

9) Mok NW, Brauer SG, Hodges PW: Hip strategy for balance control in quiet standing is reduced in people with low back pain. Spine, 2004, 29: E107E112.

10) Newcomer KL, Jacobson TD, Gabriel DA: Muscle activation patterns in subjects with and without low back pain. Arch Phys Med Rehabil, 2002, 83: 816-821. 